\title{
Efeitos da abreviação do jejum por meio de solução oral enriquecida com carboidrato (cho) em procedimentos hospitalares
}

\author{
Effects of fasting abbreviation through carbohydrate (cho) oral solution in hospital procedures
}

Efectos de la abreviatura del ayuno mediante la solución oral de carbohidratos (cho) en procedimientos hospitales

\section{Resumo}

O trabalho visa comprovar que há efeitos positivos na realização da abreviação do jejum, usando a solução enriquecida com carboidrato (CHO) em pacientes que foram submetidos a intervenções hospitalares. O principal objetivo foi mostrar se houve melhora no quadro clínico do paciente e explorar as informações já existentes na literatura. Foi feita uma revisão narrativa baseada em uma seleção dos principais artigos sobre o tema nos últimos anos, nas línguas inglesa e portuguesa. Realizou-se também uma pesquisa analítica no intuito de buscar respostas à problemática do estudo com base nos dados encontrados no Scielo, no Cochrane, no PubMe e no protocolo ACERTO para a construção desse trabalho. O resultado da pesquisa foi bastante satisfatório, mostrando que a abreviação do jejum é possível por causa da ação de uma equipe multidisciplinar e da importante ação do nutricionista ao indicar a abreviação do jejum aos pacientes. Essa redução do jejum trouxe benefícios, como, bem-estar, rápida recuperação e redução do tempo de internação dos pacientes hospitalizados.

Palavras-chave: Jejum; Pré-operatório; Carboidrato.

\begin{abstract}
The work aims to prove that there are positive effects in the abbreviation of fasting, using the solution enriched with carbohydrate $(\mathrm{CHO})$ in patients who have undergone hospital interventions. The main objective was to show whether there was an improvement in the patient's clinical condition and to explore the information already existing in the literature. A narrative review was made based on a selection of the main articles on the topic in recent years, in English and Portuguese. An analytical research was also carried out in order to seek answers to the study's problems based on the data found in Scielo, Cochrane, PubMe and the ACERTO protocol for the construction of this work. The research result was quite satisfactory, showing that the abbreviation for fasting is possible because of the action of a multidisciplinary team and the important action of the nutritionist when indicating the abbreviation of fasting to patients. This reduction in fasting brought benefits, such as, well-being, rapid recovery and reduced hospitalization time for hospitalized patients.
\end{abstract}

Keywords: Fasting; Preoperative; Carbohydrate.

\section{Resumen}

El trabajo tiene como objetivo comprobar que existen efectos positivos en la abreviatura de ayuno, utilizando la solución enriquecida con carbohidratos (CHO) en pacientes que han sido sometidos a intervenciones hospitalarias. El objetivo principal fue mostrar si hubo una mejoría en la condición clínica del paciente y explorar la información ya existente en la literatura. Se realizó una revisión narrativa a partir de una selección de los principales artículos sobre el tema en los últimos años, en inglés y portugués. También se realizó una investigación analítica con el fin de buscar respuestas al problema de estudio a partir de los datos encontrados en Scielo, Cochrane, PubMe y el protocolo ACERTO para la construcción de este trabajo. El resultado de la investigación fue bastante satisfactorio, mostrando que la abreviatura de ayuno es posible por la acción de un equipo multidisciplinar y la importante acción del nutricionista al indicar la 
abreviatura de ayuno a los pacientes. Esta reducción del ayuno trajo beneficios, como bienestar, rápida recuperación y reducción del tiempo de hospitalización para los pacientes hospitalizados.

Palabras clave: Ayuno; Preoperatorio; Carbohidratos.

\section{Introduçãa}

O Jejum é normalmente é recomendado com o propósito de obter o esvaziamento gástrico e reduzir possíveis eventos indesejados de origem respiratória devido ao bloqueio dos reflexos protetores e das vias aéreas durante o procedimento cirúrgico. Pode-se observar que o tempo de jejum nos casos cirúrgicos excede o recomendado devido a diversos fatores: atraso nas cirurgias, transferência de horário ou local de realização do procedimento. Esses fatores incentivam vários pesquisadores a procurarem alternativas para amenizar este tempo (Correia, et al., 2005; Nascimento, et al., 2016; Pierotti, et al., 2018).

Observa-se que o longo período de jejum é questionado por estudos que abordam benefícios sobre a abreviação do jejum e utilização de soluto de carboidrato antes de cirurgias. Já é possível observar que este soluto não traz malefício ao paciente, havendo, inclusive, protocolos incentivando o seu uso. O mais utilizado no Brasil é o protocolo ACERTO- ACEleração da Recuperação Total pós-operatória (Nascimento, et al., 2017).

Quando ocorre um procedimento cirúrgico, o jejum provoca alteração na homeostase do organismo, gerando um conjunto de respostas e alterações no metabolismo. Este fator é algo normal e esperado, porém, com o prolongamento do jejum pré-operatório, aumenta a resposta metabólica, gerando alterações que podem trazer complicações para o prognóstico do paciente (Marcarini, et al., 2017; Pucci, et al., 2008).

Atualmente, as recomendações do jejum sofreram modificações pelas principais sociedades de anestesia do mundo. O jejum passou a ser mais curto e foi reconhecido o fato de que a ingestão de solução oral enriquecida com carboidratos (CHO), administrado duas horas antecedentes ao procedimento cirúrgico, não traz malefícios ao paciente e nem ao procedimento exposto. Esta técnica resulta na diminuição da resistência insulínica, estresse cirúrgico, tendo melhora no bem estar do paciente, além de promover uma recuperação mais rápida (Marcarini, et al., 2017; Correia, et al., 2005).

Atualmente já se sabe que o jejum abreviado e a reintrodução precoce da dieta no Pré-operatório é fundamental para um melhor prognóstico do paciente cirúrgico, evitando intercorrências no pós-operatório e diminuindo sua estadia hospitalar (Ludwig, et al., 2013).

Dessa forma, o presente trabalho tem como objetivo apresentar os efeitos da abreviação do jejum usando a solução oral de carboidrato (CHO), demonstrando se houve melhora no quadro clínico do paciente e explorar as informações já existentes na literatura.

\section{Metodologia}

De acordo com PereiraA, et al. (2018), Trata-se de uma pesquisa descritiva, usando fontes primárias para a formação de uma revisão narrativa da literatura baseada em artigos publicados nos últimos 20 anos, em inglês e português, que abordam a abreviação do jejum e a resposta do paciente sobre o protocolo utilizado.

A busca incluiu as bases de dados PubMed, SciELO, Cochrane e o protocolo ACERTO com os descritores DeCS: Jejum, Pré-operatório, Carboidrato (Fasting; Preoperative; Carbohydrate). Dos trabalhos consultados, 41 artigos foram selecionados.

Realizou-se uma leitura analítica com o intuito de obter as respostas à problemática do estudo. As informações contidas nas fontes foram organizadas e sintetizadas de forma qualitativa, onde os resultados foram apontados em conceitos e ideias claras para a construção do manuscrito (Pereira et al., 2018).

Critérios de inclusão: Foram selecionados os artigos que abordam: métodos de abreviação de jejum em pacientes que 
foram submetidos a cirurgias com anestesia, protocolo ACERTO, tempo de jejum e solução enriquecida com carboidrato.

Critérios de exclusão: Foram excluídos os artigos em que os pacientes não precisaram de anestesia geral e que não abordam o tema.

\section{Resultados e Discussão}

A área da saúde passou por inúmeros avanços no decorrer das décadas, mas as cirurgias continuam sendo uma opção comum e eficaz para muitas doenças. E, para a realização dessas cirurgias, o paciente é informado da necessidade de realizar o jejum, que, normalmente, inicia-se na noite anterior ao procedimento (Azevedo, et al., 2017).

Durante a análise de vários artigos foi possível observar que os pacientes permanecem mais tempo em jejum do que realmente é pedido, devido a fatores como: atraso nas cirurgias; transferências, falta de profissionais e equipamentos (Chaves, et al., 2019; Nascimento, et al., 2009; Oliveira, et al., 2009; Azevedo, et al., 2017; Lobo, et al., 2009; Brianez, et al., 2014).

Callegaro, et al (2010) mostra, em seu trabalho, as experiências de alguns pacientes, como: atrasos, adiamentos ou cancelamentos. Alguns foram orientados somente sobre como deveriam se preparar para a realização da cirurgia. Em relação aos imprevistos e intercorrências, pacientes foram avisados após várias horas, gerando um aumento da expectativa e provocando malefícios de cunho psicológico para o paciente e seus familiares.

Em Actual preoperative fasting time in Brazililian hospitals: the BIGFAST multicenter study, Nascimento, et al. (2017) mostra como a situação é verídica e preocupante. No artigo foram analisados cinco estados e a conclusão aponta que $84 \%$ dos pacientes ficaram mais de 8 horas em jejum pré operatório e 25\% deles ficaram mais de 16 horas. Azevedo, et al. (2017), em suas pesquisas, encontrou resultados similares de duração de 10 a 16 horas.

Existem duas explicações para esse jejum prolongado, sendo a primeira decorrente de imprevistos gerados por falta de equipamento e superlotação (Chaves, et al., 2019). A segunda explicação, usada pelos médicos, diz que, no momento da indução anestésica, o estômago precisa estar totalmente vazio para evitar complicações respiratórias advindas de vômitos e aspiração de conteúdo gástrico. No ponto de vista metabólico esse longo tempo de jejum é extremamente prejudicial ao organismo, pois leva à depleção do estoque de glicogênio, impactando a resposta orgânica ao estresse e resultando em desconfortos como: sede, fome, resistência insulínica pós-cirúrgica e implicações na qualidade de vida e bem-estar (Azevedo, et al., 2017; Campos, et al., 2018).

Para melhorar a qualidade hospitalar para profissionais da saúde e pacientes, a estrutura e as salas de cirurgia foram mudando e se transformando, mas os cuidados perioperatórios continuaram seguindo recomendações antiquadas (Melchor, et al., 2018).

Os cuidados perioperatórios começaram a mudar com o surgimento dos "FAST TRACK", estratégias que visam melhorar o tempo de recuperação dos pacientes após a cirurgia. Essas estratégias romperam ideias tradicionais de condutas perioperatórias que normalmente são passadas de geração para geração, muitas vezes, sem a presença de evidências científicas claras (Wilmore, et al., 2001).

Em 2001 foi criado, por um grupo europeu, o ERAS — Enhanced Recovery After Surgery (Recuperação aprimorada após a cirurgia, em tradução livre) — que visa, também, diminuir o tempo de recuperação do paciente cirúrgico (Ljungqvist, et al., 2011). Foi criado para hospitais da Europa em uma condição totalmente diferente da que se observa no Brasil. Este protocolo possui um alto custo que dificulta a implantação nos países em desenvolvimento (Wilmore, et al., 2001; Vale, et al., 2019).

Visando ter maior aplicabilidade no Brasil, criou-se em 2005 o projeto ACERTO (Aceleração da Recuperação Total pós Operatória) na Universidade de Medicina de Mato Grosso (UFMT). Este projeto é uma adaptação do ERAS para a realidade de países em desenvolvimento, reduzindo, assim, os custos do protocolo e dando ênfase na terapia nutricional perioperatória. Há uma grande quantidade de pacientes desnutridos esperando para serem operados e essa condição acaba agravando muito a 
recuperação do paciente. (Nascimento, et al.,2017).

Em 2017 foi publicada a diretriz ACERTO de intervenções nutricionais no perioperatório em cirurgia geral eletiva, recomendando os profissionais a seguirem o protocolo com a alegação de que essas diretrizes aceleram a recuperação pósoperatória do paciente, o que diminui a morbidade, o tempo de internação e, consequentemente, os custos hospitalares. Como tópico desses protocolos apareceu a abreviação do jejum através de líquidos (Nascimento, et al., 2017).

No mesmo ano o CFM (Conselho Federal de Medicina) fez a resolução 2174/2017 sobre o ato anestésico, sugerindo tempos de jejum pré-operatórios semelhantes ao projeto ACERTO. A resolução contém, também, líquidos claros como: água; chá, até duas horas antes do procedimento; leite materno, em até quatro horas antes do procedimento; leite humano ou fórmulas, até 6 horas antes do procedimento. Acerca de alimentos sólidos, a recomendação foi: refeições leves, seis horas antes do procedimento; dieta normal, 8 horas antes do procedimento. Com isso, mostrou-se ética a abreviação do jejum (Brasil,cfn 2174/2017).

O uso e implantação do protocolo ACERTO é gratuito e existe uma equipe chamada Time ACERTO que ajuda na implantação deste protocolo através de seminários. O protocolo acerto visa recomendar condutas baseadas em evidências científicas de cuidados e prescrições no pré-operatório, acelerando a recuperação pós-operatória. Como exemplo de cuidado préoperatório tem-se a abreviação do estado de jejum (Nascimento, et al.,2016).

Seguindo os guidelines de anestesiologia foi possível observar que é possível abreviar o tempo de jejum pré-operatório através de líquidos sem resíduos 2 horas antes do procedimento, mostrando que bebidas ricas em CHO eram capazes de atenuar a resposta orgânica ao trauma (Oliveira, et al., 2009).

Nascimento, et al. (2006) e Bemish, et al. (2015) confirmam essa informação mostrando que, com a implementação do projeto, diminuiu-se em $70 \%$ o tempo de jejum pré-operatório, de 16 horas para 5 horas. A medicina baseada em evidências vem mostrando que programas de aceleração da recuperação pós-operatória são seguros e favorecem a diminuição de complicações pós-operatórias, reduzindo o tempo de internação hospitalar sem aumento das taxas de reinternação (Nascimento, et al., 2006).

\subsection{Importância da abreviação do jejum com o uso de líquidos originados de carboidratos.}

Para confirmar a eficácia do uso de CHO, Ludwing, et al. (2013) reuniu vários estudos que mostram que a ingestão de líquidos é segura e não gera aumento no risco de aspiração, regurgitação e mortalidade. Foram encontradas poucas ou raras complicações (incluindo regurgitação, aspiração e morbidade, sede, fome, dor, náusea, vômitos, ansiedade). Além disso, verificou-se uma diminuição da taxa de mortalidade.

O estudo de Oliveira, et al. (2009) corrobora com o de Ludwing, et al. (2013), onde, no primeiro, 365 pacientes receberam suplemento nutricional com dextrinomaltose (Polissacarídeo usado como suplemento alimentar), sendo 12,5\% antes do procedimento cirúrgico. O tempo entre a última tomada do suplemento e o início do procedimento anestésico variou entre 2 horas e 20 horas, com mediana de 4 horas e média de 3 horas. Durante a realização dos procedimentos, não se registrou nenhuma complicação relacionada ao jejum pré-operatório, como: regurgitação durante a indução anestésica ou qualquer outra complicação relacionada à abreviação do jejum para 2 horas com líquidos claros.

Oliveira, et al. (2009), não identificou risco de aspiração associada à anestesia nos estudos prospectivos e aleatórios feitos com pacientes que ingeriram bebida rica em carboidrato 2 horas antes da operação. 365 pacientes que foram submetidos à anestesia, recebendo uma solução de $400 \mathrm{ml}$ de dextrinomaltose a $12,5 \%$, seis horas antes do procedimento, e de $200 \mathrm{ml}$ duas horas antes do procedimento respectivamente, não apresentaram nenhum caso de regurgitação do conteúdo gástrico e nenhuma outra intercorrência relacionada a complicações anestésicas, não havendo aumento de risco.

Segundo os estudos apresentados por, Beamish, et al. (2015), Ludwing, et al. (2013), Nascimento, et al. (2006), Nascimento, et al. (2009), Nascimento, et al. (2016), Oliveira, et al. (2009), Power, et al. (2012), a abreviação do jejum é 
importante, pois a solução enriquecida com CHO melhora a recuperação dos pacientes. Observou-se redução nas complicações pós operatórias, infecções, mortalidade, morbidade, reoperação, tempo de internação e, principalmente, não há relato de complicações de origem respiratória.

\subsection{Implicações metabólicas do jejum no trauma cirúrgico}

A restrição de alimentos é uma prática comum utilizada pelos médicos para evitar complicações de origem aspirativas durante a realização das cirurgias, mas um tempo de jejum prolongado eleva o estresse metabólico ao trauma cirúrgico (Chaves, et al.,2019).

Ao se alimentar, o organismo transforma o carboidrato (alimento / glicose) em glicogênio através da glicogênese. Isso ocorre porque o aumento da glicose ativa o hormônio insulina, que é responsável por levar a glicose circulante no sangue para as células, iniciando o processo de glicogênese, que estoca a glicose no formato de glicogênio. O glicogênio está presente no fígado e nos músculos. Como os músculos estão em maior quantidade no organismo, o maior estoque é concentrado neles. Por isso, a proteína muscular passa a prover glicose para o fígado. Além disso, os tecidos dependem de proteína como fonte de energia, o que pode gerar uma depleção da massa muscular, em caso de jejum, podendo ser agravante na desnutrição (Guerra, 2011).

Quando se passa um longo tempo em jejum, o corpo fica sem estoque de glicose, sendo necessária a quebra do glicogênio para gerar glicose na corrente sanguínea e, assim, distribuir energia para os órgão e músculos. Neste momento os hormônios antagônicos da insulina (glucagon e cortisol) entram em ação, favorecendo a liberação da glicose sanguínea e gerando o processo de hiperglicemia (Guerra, 2011).

O paciente em jejum passa por todo esse processo de glicogenólise, gerando um estresse metabólico. Este estresse metabólico é comum, porém é potencializado em caso de jejum prolongado, o que gera malefícios para a recuperação do paciente (Chaves, et al., 2019; Nascimento, et al., 2009; Manchikanti, et al. ,2001; Medeiros, et al., 2017; Perrone, et al., 2011).

Quando o paciente passa por um trauma ou estresse dos tipos físico, metabólico, trauma ou emocional, os hormônios contra reguladores (adrenalina, noradrenalina, cortisol e glucagon) são ativados. Eles são responsáveis por aumentar a glicemuia. Quando o corpo está em estado de estresse, o cortisol induz á maior glicogênese a partir de substratos provenientes da proteólise e lipólise periférica, gerando um estado de hiperglicemia e resistência à insulina (Lawall , 2012; Medeiros, et al., 2017).

Um estado prolongado de hiperglicemia aumenta a secreção de insulina e a hipersecreção de glicocorticóides (cortisol) gera um feedback negativo para o pâncreas, que, por sua vez, inibe a secreção da insulina, gerando uma resistência à insulina $\mathrm{e}$ fazendo com o que o paciente se comporte como diabetico tipo 2 (Lawall, 2012; :Medeiros, et al., 2017; Pereira, et al., 2018).

Além da regulação dos níveis de glicose no sangue, a insulina desempenha papéis importantes na regulação imunológica e na inibição das lesões por estresse oxidativo. A insulina pode reduzir significativamente a liberação de citocinas inflamatórias e melhorar o prognóstico de pacientes criticamente enfermos. Com o paciente em resistência insulínica esse processo antiinflamatório demora mais tempo para acontecer (Chen, et al., 2020).

Observa-se que o jejum é justificado por evitar complicações de origem respiratória. Mas o elevado tempo sem alimentação muitas vezes é o responsável por gerar complicações metabólicas, torna mais lenta a recuperação do paciente e a cicatrização do paciente (Chaves, et al., 2019).

\subsection{Atuação da solução de cho no organismo}

$\mathrm{O}$ aumento da resistência insulínica e prolonga o estado de inflamação do paciente. Em decorrência desses malefícios, pesquisadores foram atrás de uma forma de amenizar essa resposta metabólica e gerar uma ação inflamatória mais controlada, melhorando, assim, a condição clínica dos pacientes (Medeiros, et al., 2017; Pereira, et al.,2018). 
Diante de várias pesquisas foi possível observar que a solução enriquecida de carboidrato age na redução da glicemia advinda de um processo inflamatório, reduzindo, consequentemente, a resistência insulínica. Isso cria um controle no aumento das alterações metabólicas, como a ação do cortisol, amenizando os traumas pré-operatórios. A diminuição da resistência insulínica pode ser explicada mostrando que o carboidrato aumenta a capacidade da glicose em regular a resposta metabólica e inflamatória. Com isso, diminui-se o tempo de internação e melhora a resposta do paciente, tornando mais rápida a introdução da alimentação oral e, consequentemente, a melhora clínica do paciente (Diógines, et al., 2019; Guerra, 2011; Nascimento, et al., 2007; Nascimento, et al.,2017).

Para que todos esses benefícios aconteçam, vários profissionais participam do preparo e do cuidado do paciente antes e após a cirurgia. O nutricionista é responsável por avaliar o estado nutricional do paciente e seu risco de desnutrição. Se necessário, o profissional age melhorando as condições nutricionais dos pacientes para evitar as complicações do estresse metabólico, como a perda de massa muscular e desnutrição (Chaves et al., 2019; Guerra ,2011; Nascimento, et al., 2017; Merhi, et al., 2000).

Carvalho, et al. (2019), relata que o uso de carboidrato traz benefícios como melhora no bem estar, metabolismo glicêmico, diminuição da resistência insulínica, menor perda de massa magra e uma melhora na recuperação. Em seu estudo, foi oferecido aos pacientes $12,5 \%$ de maltodextrina diluída em $150 \mathrm{ml}$ de água duas horas antes do procedimento.

No trabalho avaliram- se principalmente, os exames bioquímicos, onde encontrou-se um valor menor de Proteína C reativa (proteína produzida pelo fígado, onde sua concentração sanguínea se eleva quando existe um processo inflamatórios). Os valores de albumina apresentaram-se elevados em pacientes ingeriram CHO. Estes resultados, PCR baixo e albumina elevada, trouxeram um bom prognóstico para o paciente, pois quando a albumina está baixa apresenta-se um prognóstico ruim no paciente, normalmente significativo de desnutrição, já o PCR elevado demostra inflamação, sendo assim outro valor que traz uma visão negativa aos resultados do paciente (Carvalho, et al.,2019).

\subsection{Esvaziamento gástrico e segurança da abreviação do jejum}

Pesquisadores relatam que o uso da solução contendo carboidrato não gerou complicações, além de não haver alteração do PH gástrico, considerando, por fim, o procedimento seguro (Carvalho, et al., 2019; Feguri ,et al., 2012).

A literatura indica que soluções com o volume variado, mas com a mesma quantidade de carboidrato, compreendem resultados de esvaziamento gástrico semelhantes apontando que a solução depende necessariamente da presença de nutrientes e não do volume (Campos, et al., 2018).

Considerando fatores envolvidos no processo de esvaziamento gástrico, estudos vêm sendo produzidos com o objetivo de esclarecer a segurança da abreviação do jejum (Brianez, et al., 2014; Awad, et al., 2011).

Chaves, et al. (2019), relacionou alguns estudos sobre o uso do CHO. Observou que não há relatos de intercorrências advindas do uso da solução durante o procedimento. Foi possível observar melhorias no período pré-operatório como: redução de desconforto abdominal, redução no tempo de internação, diminuição da fase aguda referente ao trauma, aumento dos níveis de antioxidantes, redução do hormônio cortisol, manutenção da massa corporal muscular, diminuição da proteína C reativa.

Notou-se que é consenso na maioria dos estudos que a abreviação do jejum com bebidas ricas em CHO até duas horas antes do procedimento cirúrgico é segura e apresenta benefícios para o paciente. Diminui a resistência insulínica e o estresse metabólico, além de contribuir significativamente para o bem-estar do paciente, reduzir o tempo de hospitalização e, consequentemente, os custos da internação (Chaves, et al., 2019; Nascimento, et al., 2012; Pimenta, et al., 2013).

\subsection{Papel do nutricionista na abreviação do jejum}

O projeto ACERTO avançou muito na parte de nutrição pré-operatória. Atualmente todos os pacientes que passam por esse projeto são submetidos a uma triagem nutricional. Os pacientes desnutridos, ou que tiveram risco de desnutrição, ou 
que serão submetidos a cirurgias de grande porte, devem se submeter à terapia nutricional pré-operatória com o objetivo de diminuir os riscos de complicações pós-operatórias. Devem, ainda, passar por terapia nutricional contínua no pós-operatório (Nascimento, et al., 2017).

O nutricionista é um dos profissionais responsáveis por garantir que o protocolo seja seguido. Ele auxilia em: não permitir um jejum prolongado, indicar o uso de dieta líquida enriquecida com carboidrato na véspera da operação, acompanhar o pós operatório e prescrever a melhor dieta para cada procedimento específico. Ele é quem prescreve a dieta pré e pós operatória para cada paciente, averiguando, juntamente com a Equipe Multiprofissional de Terapia Nutricional (EMTN) do hospital, há quanto tempo o paciente está em jejum, quais comorbidades ele apresenta e qual protocolo deve-se seguir no pós-operatório (Martins, et al., 2020; Nascimento, et al., 2006; Oliveira, et al., 2009 ).

A indicação do protocolo de abreviação de jejum deve ser feita antes mesmo da internação, informando o paciente acerca do assunto. Faz-se necessária a padronização deste tipo de protocolo em todos os hospitais, visando menor tempo de internação. (Nascimento, et al., 2016).

Está nas mãos, principalmente dos nutricionistas, a constância do manuseio do protocolo de abreviação do jejum, sendo a maior influência dentro do hospital para que médicos também adotem em sua totalidade (Nascimento, et al., 2006).

\section{Considerações Finais}

Com o intuito de estudar os efeitos da abreviação do jejum usando a solução oral de CHO. Realizou-se uma revisão de literatura, selecionando os artigos científicos que melhor abordaram o tema.

Pode-se, portanto, observar que o protocolo ACERTO incentivou a diminuição no tempo de jejum pré-operatório, mostrando benefícios na melhora na recuperação do paciente. Evidenciando que a abreviação do jejum resulta na diminuição da resistência insulínica gerada pelo estresse cirúrgico, havendo uma melhora significativa do bem-estar do paciente, promovendo uma recuperação mais rápida e reduzindo o tempo de internação.

A abreviação do jejum é possível por consequência de uma equipe multidisciplinar voltada para os cuidados do paciente e o nutricionista é o profissional capacitado para avaliar o estado nutricional do paciente e prescrever a abreviação do jejum.

Entretanto, a implementação do protocolo de abreviação de 2 a 3 horas de jejum acaba sendo um dos maiores desafios atuais. É necessário promover mudanças de condutas, reeducando toda a equipe de saúde que realiza os protocolos com o paciente durante a internação hospitalar.

Os estudos utilizados como base para o desenvolvimento deste trabalho mostraram-se eficientes e eficazes na abreviação do jejum com o uso de solução oral enriquecida de carboidrato (CHO). Os diversos autores pesquisados chegaram à conclusão de que a abreviação do jejum é segura, diminui a mortalidade, tempo de internação e melhora a qualidade de vida do paciente.

Este tema mostra-se muito importante e relevante para a saúde e recuperação do paciente, portando é necessário o desenvolvimento de mais artigos sobre abreviação do jejum e abordando de forma mais detalhada como o organismo humano reage a esta abreviação e a não abreviação.

\section{Referências}

Awad, S., Blackshaw, P. E., Wright, J. W., Macdonald, I. A., Perkins, A. C., \& Lobo, D. N. (2011). A randomized crossover stu dy of the effects of glutamine and lipid on the gastric emptying time of a preoperative carbohydrate drink. Clinical nutrition 30(2), $165-171$.

Azevedo, S. C. L., Campos, S. B. G., de Meira, J. E. C., \& da Silva Guedes, G. (2017). Abreviação do jejum pré-operatório: protocolo multimodal baseado em evidência. Gep News, 1(3), 11-13. 
Beamish, A. J., Chan, D. S. Y., Blake, P. A., Karran, A., \& Lewis, W. G. (2015). Systematic review and meta-analysis of enhanced recovery programmes in gastric cancer surgery. International Journal of Surgery, 19, 46-54.

Brianez, L. R., Caporossi, C., de Moura, Y. W., Dias, L. A., Leal, R. V., \& Aguilar-Nascimento, J. E. (2014). Gastric residual volume by magnetic ressonance after intake of maltodextrin and glutamine: a randomized double-blind, crossover study. Arquivos de gastroenterologia, 51(2), 123-127.

Callegaro, G. D., Baggio, M. A., Do Nascimento, K. C., \& Erdmann, A. L. (2010). Cuidado perioperatório sob o olhar do cliente cirúrgico. Revista da Rede de Enfermagem do Nordeste, 11(3), 132-142.

Campos, S. B. G., Barros-Neto, J. A., Guedes, G. D. S., \& Moura, F. A. (2018). Jejum Pré-operatório: Por que Abreviar? ABCD. Arquivos Brasileiros de Cirurgia Digestiva, 31(2).

Carvalho, C. A. L. D. B., Carvalho, A. A. D., Preza, A. D., Nogueira, P. L. B., Mendes, K. B. V., Dock-Nascimento, D. B., \& Aguilar-Nascimento, J. E. (2020). Benefícios Metabólicos e Inflamatórios da Abreviação do Jejum Pré-operatório em Cirurgia Pediátrica. Revista do Colégio Brasileiro de Cirurgiões, 47.

Chaves, L. M., \& Prazeres Campos, J. S. (2019). Abreviação do jejum e suporte nutricional pré-operatório em cirurgias eletivas: Uma revisão sistemática. Revista Eletrônica Acervo Saúde, (35), e2210-e2210.

Chen, Q., Yu, W., Shi, J., Shen, J., Gao, T., Zhang, J., \& Li, N. (2014). Insulin alleviates the inflammatory response and ox idative stress injury in cerebral tissues in septic rats. Journal of Inflammation, $11(1), 18$.

Correia, M. I. T. D., \& Silva, R. G. D. (2005). Paradigmas e evidências da nutrição peri-operatória. Revista do Colégio Brasileiro de cirurgiões, 32(6), 342347.

Diógenes, D. H., Costa, C. S. D., \& Rivanor, R. L. D. C. (2019). Tempo de Jejum no Pré-Operatório de Cirurgias Eletivas em um Hospital de Referência em Trauma no Município de Fortaleza CE. Rev. bras. ciênc. saúde, 191-196.

Feguri, G. R., Lima, P. R. L., Lopes, A. M., Roledo, A., Marchese, M., Trevisan, M., ... \& de Aguilar-Nascimento, J. E. (2012). Resultados clínicos e metabólicos da abreviação do jejum com carboidratos na revascularização cirúrgica do miocárdio. Revista Brasileira de Cirurgia Cardiovascular/Brazilian Journal of Cardiovascular Surgery, 27(1), 7-17.

Guerra, R. (2011). Cadernos Cb Virtual 2.

Lawall,T (2012). Seminário da disciplina transtornos metabólicos dos animais domésticos- diabtes mélitus, Pós graduação em ciências Veterinárias

Ljungqvist, O. (2011). ERAS-enhanced recovery after surgery. Journal de chirurgie viscérale, 148(3), e157-9.

Lobo, D. N., Hendry, P. O., Rodrigues, G., Marciani, L., Totman, J. J., Wright, J. W., \& Fearon, K. C. (2009). Gastric emptying of three liquid oral preoperative metabolic preconditioning regimens measured by magnetic resonance imaging in healthy adult volunteers: a randomised double-blind, crossover study. Clinical Nutrition, 28(6), 636-641.

Ludwig, R. B., Paludo, J., Fernandes, D., \& Scherer, F. (2013). Menor tempo de jejum pré-operatório e alimentação precoce no pós-operatório são seguros? ABCD. Arquivos Brasileiros de Cirurgia Digestiva (São Paulo), 26(1), 54-58.

Manchikanti, L., Malla, Y., Wargo, B. W., \& Fellows, B. (2011). Preoperative fasting before interventional techniques: Is it necessary or evidence-based. Pain Physician, 14(5), 459-67.

Marcarini, M., Rosa, S. C. D., Wieck, F. P., \& Betti, A. H. (2017). Abreviação do jejum: aspectos clínicos perioperatórios de pacientes submetidos à cirurgia cardíaca. Braspen J, 375-379.

Martins, M. S., de Castro, G. P., Santos, K. P. C., Fontenele, F. F., \& de Sousa Rocha, V. (2020). Tempo de jejum, estado nutricional e complicações pósoperatórias de pacientes submetidos a cirurgias eletivas. Research, Society and Development, 9(7), e53973646-e53973646.

Medeiros, A. C., \& Dantas Filho, A. M. (2017). Resposta metabólica ao trauma. Journal of Surgical and Clinical Research, 8(1), 56-76.

Melchor, J., Varela, M. L. D. F., Camargo, S. C., Fernández, P. J., Barrio, Á. C. D., Martínez-Hurtado, E., \& Calvo-Vecino, J. M. (2018). Enhanced recovery after surgery protocol versus conventional perioperative care in colorectal surgery. A single center cohort study. Revista brasileira de anestesiologia, 68(4), 358368.

Merhi, V. A., Garcia, R. W. D., Tafner, B., Florentino, M. C., Casteli, R., \& de Aquino, J. L. B. (2012). Relação entre o estado nutricional e as características clínicas de pacientes internados em enfermarias de cirurgia. Revista de Ciências Médicas, 9(3).

Nascimento, D. B., de Aguilar-Nascimento, J. E., Magalhaes Faria, M. S., Caporossi, C., Slhessarenko, N., \& Waitzberg, D. L. (2012). Evaluation of the effects of a preoperative 2-hour fast with maltodextrine and glutamine on insulin resistance, acute-phase response, nitrogen balance, and serum glutathione after laparoscopic cholecystectomy: a controlled randomized trial. Journal of Parenteral and Enteral Nutrition, 36(1), 43-52.

Nascimento, J. E. (2016). ACERTO-Acelerando a recuperação total pós-operatória. Editora Rubio.

Nascimento, J. E. D., Bicudo-Salomão, A., Caporossi, C., Silva, R. D. M., Cardoso, E. A., \& Santos, T. P. (2006). Acerto pós-operatório: avaliação dos resultados da implantação de um protocolo multidisciplinar de cuidados peri-operatórios em cirurgia geral. Revista do Colégio Brasileiro de Cirurgiões, 33(3), 181-188.

Nascimento, J. E. D., Dock-Nascimento, D. B., Faria, M. S. M., Maria, E. V., Yonamine, F., Silva, M. R., \& Adler, T. (2007). Ingestão pré-operatória de carboidratos diminui a ocorrência de sintomas gastrointestinais pós-operatórios em pacientes submetidos à colecistectomia. ABCD. Arquivos Brasileiros de Cirurgia Digestiva (São Paulo), 20(2), 77-80. 
Research, Society and Development, v. 10, n. 1, e13010111580, 2021

(CC BY 4.0) | ISSN 2525-3409 | DOI: http://dx.doi.org/10.33448/rsd-v10i1.11580

Nascimento, J. E. D., Perrone, F., \& Assunção Prado, L. Í. D. (2009). Jejum pré-operatório de 8 horas ou de 2 horas: o que revela a evidência? Revista do Colégio Brasileiro de Cirurgiões, 36(4), 350-352.

Nascimento, J. E., de Almeida Dias, A. L., Dock-Nascimento, D. B., Correia, M. I. T., Campos, A. C., Portari-Filho, P. E., \& Oliveira, S. S. (2014). Actual preoperative fasting time in Brazilian hospitals: the BIGFAST multicenter study. Therapeutics and Clinical Risk Management, 10, 107.

Nascimento, J. E., Salomão, A. B., Waitzberg, D. L., Dock-Nascimento, D. B., Correa, M. I. T., Campos, A. C. L., \& Caporossi, C. (2017). Diretriz ACERTO de intervenções nutricionais no perioperatório em cirurgia geral eletiva. Revista do Colégio Brasileiro de Cirurgiões, 44(6), 633-648.

Oliveira, K. G. B. D., Balsan, M., Oliveira, S. D. S., \& Aguilar-Nascimento, J. E. (2009). A abreviação do jejum pré-operatório para duas horas com carboidratos aumenta o risco anestésico? Revista Brasileira de Anestesiologia, 59(5), 577-584.

Pereira, A. A., Rezende C., D., Santana, S., I., A., Santana, C., L., \& Amaral, B., A., P. (2018). Relação entre o hormônio cortisol e a síndrome metabólica. Seminário Estudantil de Produção Acadêmica, 17.

Pereira, A. S. et al. (2018). Metodologia da pesquisa científica. UAB/NTE/UFSM

Perrone, F., da-Silva-Filho, A. C., Adôrno, I. F., Anabuki, N. T., Leal, F. S., Colombo, T., \& de Aguilar-Nascimento, J. E. (2011). Effects of preoperative feeding with a whey protein plus carbohydrate drink on the acute phase response and insulin resistance. A randomized trial. Nutrition journal, $10(1), 66$.

Pierotti, I., Nakaya, T. T., Garcia, A. K. A., do Nascimento, L. A., Conchon, M. F., \& Fonseca, L. F. (2018). Avaliação do tempo de jejum e sede no paciente cirúrgico. Revista Baiana de Enfermagem32,

Pimenta, G. P., \& de Aguilar-Nascimento, J. E. (2014). Prolonged preoperative fasting in elective surgical patients: why should we reduce it? Nutrition in Clinical Practice, 29(1), 22-28.

Power, S., Kavanagh, D. O., McConnell, G., Cronin, K., Corish, C., Leonard, M., \& Connolly, J. (2012). Reducing preoperative fasting in elective adult surgical patients: a case-control study. Irish journal of medical science, 181(1), 99-104.

Pucci, N. D., Fontes, B., \& Poggetti, R. S. (2008). Avaliação de um esquema de realimentação utilizado após 43 dias de jejum voluntário. Revista de Nutrição, 21(5), 503-512.

Resolução N 2147/2017 Fevereiro de 2018. Dispõe sobre aprática do ato anestésico e revoga a Resolução CFM nº 1.802/2006, Brasília, DF, fevereiro 2018.

Vale, N., \& do Vale, A. H. B. ERAS (Enhanced Recovery after Surgery): um Programa para aumentar a Segurança de Procedimentos Cirúrgicos de Grande Porte. Revista potiguar de anestesiologia, 59056, 78.

Wilmore, D. W., \& Kehlet, H. (2001). Management of patients in fast track surgery. Bmj, 322(7284), 473-476. 